\title{
Mechanical Milling Of Copper Oxide Nanoparticles
}

\author{
B. Geetha Priyadarshini \\ \{bgp@psgias.ac.in\} \\ Nanotech Research Innovation and Incubation Centre, PSG Institute of Advanced Studies, Peelamedu \\ Coimbatore- 641004, Tamil Nadu, India.
}

\begin{abstract}
We describe a mechanical ball milling technique for producing $\mathrm{CuO}$ nanoparticles. Using a 10:1 ball to powder ratio and a milling speed of $150 \mathrm{rpm}$ for 20 hours, copper oxide powder was created. The X-ray diffraction (XRD) confirmed a high degree of crystalline and the average crystallite size was determined using the Scherer equation. EDS was used to determine the nanoparticles' elemental composition. EDS confirmed the purity of $\mathrm{CuO}$ nanoparticles. Dynamic light scattering was used to estimate particle size distribution. Milled copper oxide nanoparticle thermal stability and energy gap were also studied.
\end{abstract}

Keywords: Mechanical milling, copper oxide nanoparticles.

\section{Introduction}

$\mathrm{CuO}$ nanoparticles are being studied for their unique thermal and mechanical characteristics. The ball mill is a top-down method for creating ultrafine particles. Produit from severe plastic deformation of coarse granules. Top down techniques such as high intensity ball milling have been extensively used to synthesise nanomaterials, nanograins, nanoalloys, and nanocomposites. The particle deformation and fracture process that happens as a consequence of multiple ball-powder impact events allows the production of nanocrystalline structures in as-milled powder. Chemical reactions occur at the nanoscale grain surfaces, which renew during milling. Grinding balls smash and tear the material in two or four grinding bowls. The centrifugal forces operate on the grinding balls and the material in the grinding bowl owing to the grinding bowl's rotation and the spinning supporting disc. The grinding bowl and the supporting disc revolve in opposing directions, alternating centrifugal forces. As a consequence of friction, the grinding balls run down the inner wall of the bowl, and hit against the opposing wall.

\subsection{Mechanical Alloying}

Mechanical alloying is the process of milling two pure metal powders together to generate a solid solution, intermetallic or amorphous phase. Material transfer is required to generate a homogeneous alloy. High-energy milling flattens, fractures, and rewelds powder particles. 


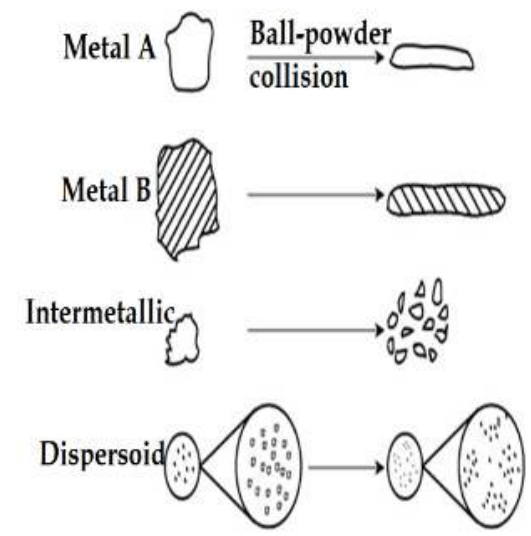

Fig: 1 Deformation characteristics of representative constituents of starting powders in mechanical alloying [1]

Fig 1 shows the consequences of a single impact on each kind of powder particle. Flattening and hardening ductile metal particles produces the grinding ball effect. Extreme plastic deformation enhances particle surface to volume ratio and ruptures adsorbed contaminating surface coatings. Fractured and refined intermetallic brittle powder particles The oxide dispersion particles are finer. Mechanical alloying permits the alloying of elements that are difficult or impossible to melt together. Alloying is used to improve strength, corrosion resistance, or cost. When two grinding balls hit, a little powder is stuck between them. Each impact traps roughly 1000 particles weighing around 0.2 milligrammes (Fig 2).

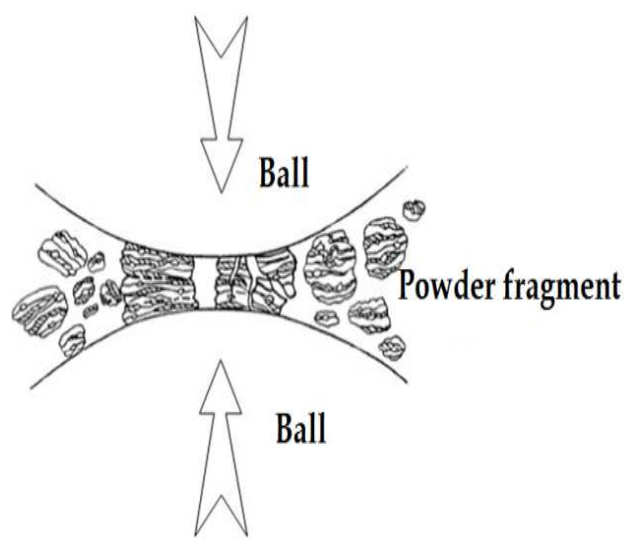

Fig: 2 Ball-powder-ball collision of powder mixture during mechanical alloying [1] 


\subsection{Mechanical Milling}

Mechanical Milling is used to homogenise powders of homogenous composition, such as pure metals intermetallics, or prealloyed powders, without material transfer. Mechanical milling is usually done using a planetary ball mill. A refractory or steel ball's energy transmission to a powder relies on the rotating (vibration) speed, size and quantity of balls, ball-to-powder mass ratio, duration and milling environment [2]. The shear action of grinding produces nanoparticles. During milling, powder particles are caught between colliding balls and deformed or fractured, defining their final structure. The nature of these processes relies on the powder components' mechanical behaviour, phase equilibrium, and milling stress state.

\subsection{Mechanochemical Milling}

Chemical reactions and phase changes occur during milling in mechanochemical processing (MCP). The capacity to store and focus stress energy is required to initiate a mechanochemical reaction. A high-energy shaker or ball mill is used in Mechanochemical Synthesis [3]. Mechanical energy used in treatment lowers reactant diffusion lengths through shear deformation, fracture, cold welding, and agglomeration. This dispersion and mixing of reagents enhances the creation of new phases by minimising the importance of size variables in diffusion and phase formation [4].

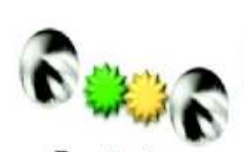

Reactants

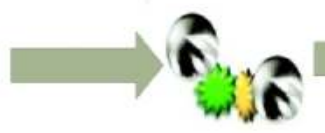

Elastic deformation

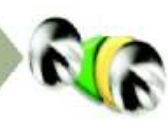

Plastic deformation

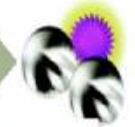

Chemical reaction

Fig: 4 Schematic illustration of mechanochemical reaction [3]

\section{Experimental Procedure}

15g cupric oxide (Sigma Aldrich, 98\%) with 15g alumina (Sigma Aldrich, 99 percent purity). Stainless steel vials and balls are utilised here. $15 \mathrm{~g}$ copper oxide powder and $15 \mathrm{~g}$ alumina powder were taken. Milling was done using stainless steel vials and $3 \mathrm{~mm}$ stainless steel balls at a 10:1 ball to powder ratio. Then, after 20 hours of milling at 150rpm, the copper oxide nanoparticles were created (45 mins cooling period for each 45 mins milling). The samples were made in a dry medium with an air environment. 


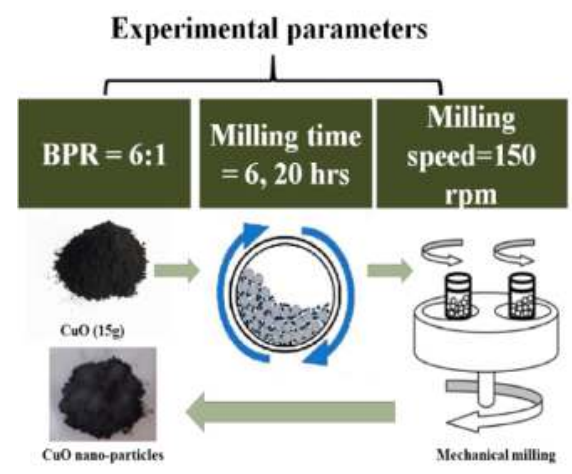

\subsection{INSTRUMENTS}

In the 3rd generation Empyrean Malvern Panalytical multifunctional diffractometer with Multi Core Optics. The X-ray source is $\mathrm{Cu} \mathrm{K}$ with a wavelength of 1.54. The SEM EVO 18 model acquired the energy dispersive X-ray spectrometry (SEM). DLS was used to determine the particle size distribution of the oxide powders suspended in water using a Nanosizer N4 (Beckman Coulter) and a Zetasizer Nano S ZEN1600 (Malvern Instruments) with movable lens and $623.8 \mathrm{~nm}$ laser beam. The simultaneous thermal analyzer (TGA-DSC) used was the Netzsch STA449 F3 Jupiter, and the spectrophotometer used was the Shimadzu UV-1800

\section{Results Anddiscussion}

X-ray diffraction (XRD) is a potent nondestructive crystalline material characterisation method. Structure, phase, preferred orientation (texture), and other structural characteristics including average grain size, crystalline, strain, and crystal defects [5] are all provided

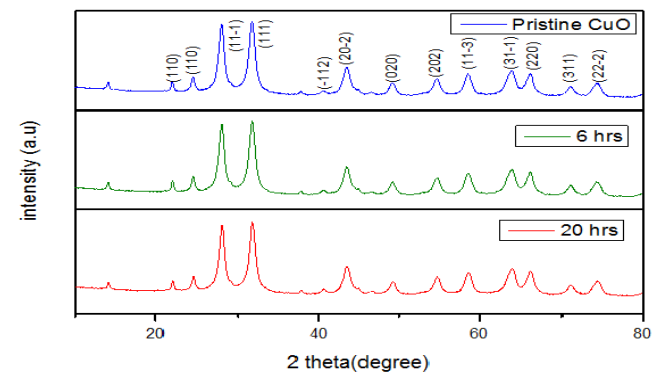

Fig: 6. XRD patterns of pristine, $6 \mathrm{hrs}$ and $20 \mathrm{hrs}$ milled $\mathrm{CuO}$ nanoparticles synthesized by ball milling method

The $6 \mathrm{hrs}$ and $20 \mathrm{hrs}$ milled copper oxide nanoparticles were illuminated by powder X-ray diffraction. (11-3), (31-1), (220), (311) and (20-2) were seen (22-2). These peaks 
identified $\mathrm{CuO}$ nanoparticles. The acquired XRD patterns matched those in the JCPDS database file no: 01-077-7716. The results matched the literature (29). Figure 6 shows the $\mathrm{XRD}$ spectrum of $\mathrm{CuO}$ nanoparticles.

The tiniest crystallite is a single crystal in powder form. XRD was then used to estimate crystallite size. The crystallite size is estimated using the corrected diffraction profile's full width at half maximum intensity (FWHM). Table 1 shows the average size of pristine, 6 and 20 hour milled $\mathrm{CuO}$ NPs.

Table: 1 Average size of pristine, $6 \mathrm{hrs}$ and $20 \mathrm{hrs}$ milled CuO NPs

\begin{tabular}{|l|l|}
\hline Copper Oxide NPs & Average Crystallite size \\
\hline Pristine $\mathrm{CuO}$ & $57 \mathrm{~nm}$ \\
\hline $6 \mathrm{hrs}$ milled $\mathrm{CuO}$ & $54 \mathrm{~nm}$ \\
\hline $20 \mathrm{hrs}$ milled $\mathrm{CuO}$ & $46 \mathrm{~nm}$ \\
\hline
\end{tabular}

Energy dispersive X-rays spectrometry (EDS) was performed to analyze the elemental constituent of the $20 \mathrm{hrs}$ milled copper oxide nanoparticles.

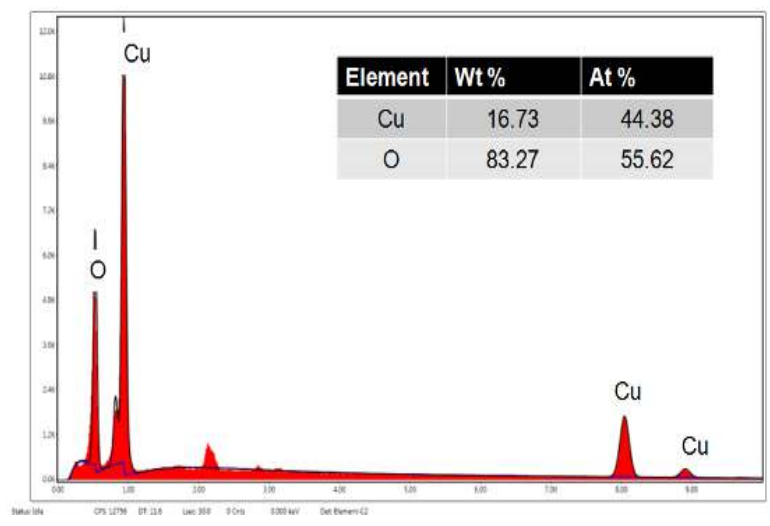

Fig: 7. EDS pattern of CuOnanoparticles milled for $20 \mathrm{hrs}$ (inset : Elemental analysis)

EDS demonstrated the purity of $\mathrm{CuO}$ NPs. Oxygen and copper in the EDS spectra suggest copper oxide or dioxide. Copper $(\mathrm{Cu})$ was 83.27 percent and oxygen (16.73 percent) was 16.73 percent. The atomic compositions were then determined to be 55.62 and 44.38 percent.

DLS was used to analyse the oxide particle size distribution. Figure 8 displays polydispersed $\mathrm{CuO}$ with two separate size populations. $\mathrm{CuO}$ aggregates are mostly micrometric, with a tiny but considerable percentage being nanometric 


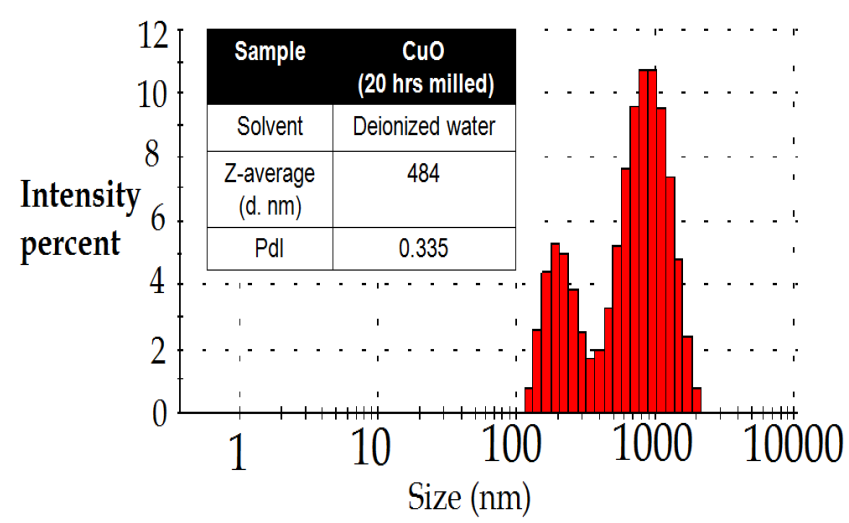

Fig: 8. DLS of CuOnanoparticles milled for $20 \mathrm{hrs}$

DLS tests showed particle sizes of $484 \mathrm{~nm}$. The DLS findings showed the purity of CuO NPs. O2 and $\mathrm{Cu}$ in EDS spectra indicate copper oxide or dioxide. Copper $(83.27 \%)$ and oxygen $(16.73 \%)$ So, the atomic compositions were 55.62 and 44.38 percent.

Dielectrophoresis (DLS) was utilised to study oxide 8 shows polydispersed $\mathrm{CuO}$ with two size populations. $\mathrm{CuO}$ aggregates are predominantly micrometric, with some nanometric.

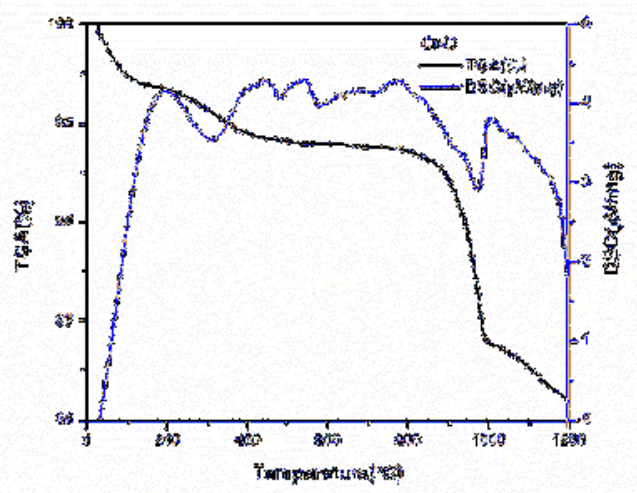

Thermal stability of $\mathrm{CuO}$ nanoparticles were investigated by Thermogravimetric analysis and Differential Scanning Calorimetry ((TGA-DSC) at a heating rate of $10^{\circ} \mathrm{C} / \mathrm{min}$ in air over a temperature range of $25-1200^{\circ} \mathrm{C}$. 


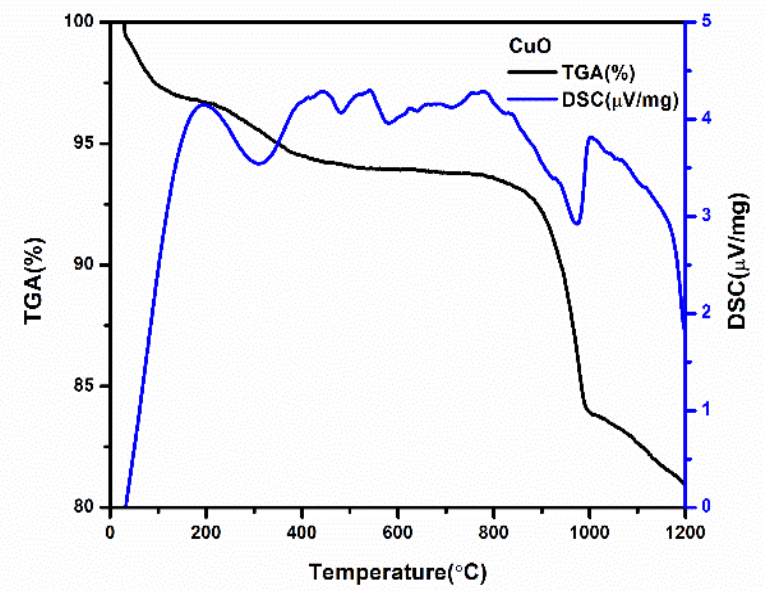

Fig:9. TGA-DSC pattern of $\mathrm{CuO}$ milled for $20 \mathrm{hrs}$

FIG. 9: $\mathrm{CuO}$ nanoparticles 20-hour TGA curve Water evaporation generated a 4\% weight loss between 25 and $200^{\circ} \mathrm{C}$, whereas oxide ion disintegration caused a $15 \%$ weight loss between $200-1200^{\circ} \mathrm{C}$.

UV-vis spectroscopy on $\mathrm{CuO}$ nanoparticles was used (Fig.10). The scan distance was 200-800 nm. Using Tauc's approach, the absorbance vs wavelength charts revealed a $3.8 \mathrm{eV}$ band gap energy. The Tauc figure shows (hv)2. The band gap energies of $\mathrm{CuO}$ nanoparticles.

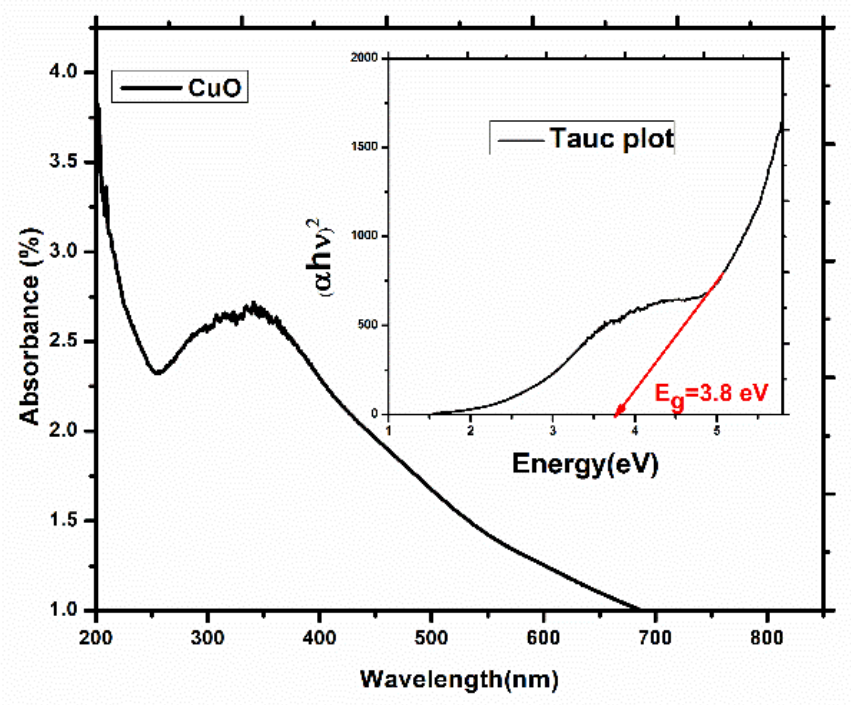

Fig: 10. UV-vis absorption spectrum of the $\mathrm{CuO}$ nanoparticles (20 hrs milled)

The energy gap from VB to CB calculated by extrapolation at the rapid decline of the Tauc's plot with substantial absorption. The second band gap at lower energy is caused by the activator, inherent defect, 
or secondary phase. Unusually large surface plasmon resonance peak at $300 \mathrm{~nm}$ may be indicative of these nanoparticles in deionized water. The rise in the band gap of $\mathrm{CuO}$ indicates the existence of tiny nanoparticles. An inherent flaw or secondary phase existing in the band gap area causes the secondary hump found in clean samples.

\section{Conclusions}

This article introduced the mechanical ball milling Using cupric oxide as a precursor, make $\mathrm{CuO}$ nanoparticles. Mechanical ball milling has cheap cost, tiny particle size, narrow size dispersion, and homogeneous crystal structure and morphology. The $\mathrm{CuO}$ nanoparticles were characterised by UV-Vis, EDS, DLS, TG-DSC, and XRD. The findings show that high energy ball milling can easily manufacture nanoparticles in commercial quantities using low-cost equipment.

\section{References}

[1] Suryanarayana, C., 2019. Mechanical alloying: a novel technique to synthesize advanced materials. Research,2019.

[2] Alagarasi, A., 2013. Chapter-Introduction to Nanomaterials. Indian Institute of Technology Madras, pp.1-24.

[3] Xu, C., De, S., Balu, A.M., Ojeda, M. and Luque, R., 2015. Mechanochemical synthesis of advanced nanomaterials for catalytic applications. Chemical communications, 51(31),pp.66986713.

[4] Ivanov, E. and Suryanarayana, C., 2000. Materials and process design through mechanochemical routes. Journal of materials synthesis and processing, 8(3),pp.235-244.

[5] Birks, L.S. and Friedman, H., 1946. Particle size determination from X-ray line broadening. Journal of Applied Physics, 17(8),pp.687-69

[6] D S Vijayan, S.Arvindan, D.Parthiban, B.Saravanan, M kalpana, "Natural aggregates used for Light weight concrete - A Review", IOP Conf. Series: Materials Science and Engineering, 993 (2020) 012042, doi:10.1088/1757-899X/993/1/012042 